\title{
Televisión Digital Interactiva. Experiencias hacia una comunicación transmedia
}

institucional.us.es/ambitos/

\author{
Marián Alonso González \\ Universidad de Sevilla \\ malonsog@us.es \\ María José García Orta \\ Universidad de Sevilla \\ mjorta@us.es
}

English Version: Digital Interactive Television. Experiences towards a communication transmedia.

\section{Resumen}

Este artículo se enfoca en torno a una revisión hemerográfica sobre la evolución que ha experimentado la interactividad en el ámbito audiovisual en España. Desde

las primeras experiencias asociadas a las cadenas privadas hasta los actuales modelos en los que se otorga el control de la emisión a los usuarios, muchas han sido las posibilidades de interacción facilitadas por internet. Del análisis realizado se concluye que el paradigma moderno de la convergencia digital hacia una televisión interactiva implica que el modelo esté virando hacia una comunicación transmedia que traslada un mensaje integrado a través de múltiples medios, lenguajes y


plataformas.

\section{Palabras clave}

Televisión, interactiva, audiencia, transmedia, digital.

Abstract

This paper focuses on a review about the evolution that has experienced the interactivity in the Spanish audiovisual area. From the first experiences associated to the private channels, just the current models that give the control of the emission for the viewers who help to do the composition of schedules of television programmes, many are the opportunity for interaction provided by internet. The study of the television experiences stands out

how the relational paradigms between production and receipt towards an interactive television implies change towards the communication transmedia that seeks to move a integrated message across languages and platforms.

\section{Keywords}

Television, interactive, audience, transmedia, digital.

\section{INICIOS DE LA TELEVISIÓN INTERACTIVA EN ESPAÑA}

La interactividad en el ámbito audiovisual no es un fenómeno nuevo en España. De hecho, desde la aparición de la televisión digital el sector siempre ha estado a la expectativa de lo que parecía ser la revolución del medio. Sin embargo, los servicios avanzados asociados a la televisión se comenzaron a vislumbrar en España en la década de los 90, de la mano del Teletrebol de Telecinco.

Según Joaquín Pérez (2011) el Teletrebol, en servicio en 1992, estaba programado para que cuando se sucediese una combinación numérica que coincidía con las respuestas correctas, se encendiera una luz y sonara el Himno de la Alegría. Era entonces cuando el espectador debía llamar a un número de teléfono 
Premium, con contestador, pegar el mando al teléfono, apretar un botón que emitía una señal que reconocía el sistema telefónico y dejar sus datos personales. Entre los afortunados, Telecinco sorteaba premios menores, fiches de semana en casas rurales, etc. Sin embargo, terminó en los tribunales por varias demandas de los espectadores, lo que provocó que la cadena lo cancelara inmediatamente.

El Teletrebol fue sustituido ese mismo año por Hugo, un troll de animación que debía rescatar a Hugolina y sus hijos de la bruja Maldicia. Se trataba del primer videojuego interactivo puesto en marcha por una televisión en España y que permitía a los espectadores participar a través del teléfono. El juego constaba de tres fases: el tren, el avión y el rescate final. Durante el juego se conseguía una puntuación que luego se traducía en pesetas, pudiéndose ganar, como máximo, 300.000 pesetas (1.800 euros).

Tan sólo un año después, en 1993, surge el Telepick, el primer dispositivo receptor, con capacidades de impresión, que permitía a los usuarios interactuar con los programas de televisión, sobre todo con los concursos, a los que se podían enviar las respuestas mediante el mando a distancia.

El telepick, que tuvo su periodo de adaptación y prueba durante las retransmisiones asociadas a la Exposición Universal de Sevilla y los Juegos Olímpicos de Barcelona de 1992, fue lanzado al mercado a un precio de 22.000 pesetas (132 euros) y para su uso tan sólo se requería una conexión de datos: un módem que se conectaba a la red telefónica y ocupaba la línea mientras el aparato estaba funcionando.

Su escasa tasa de penetración (sólo 13.000 unidades vendidas), unido a una reducida integración en el sistema de la programación de TVE, cuya intención inicial pasaba por la inclusión del servicio en el $70 \%$ de su parrilla, sobre todo en películas, programas y retransmisiones deportivas, provocó que quince meses después de su lanzamiento se produjera la quiebra de la empresa que lo había llevado al mercado (Interactive Television) y el cese del servicio.

Con la llegada del nuevo siglo y de la mano de Canal Satélite Digital y Vía Digital, que ofrecían varios servicios que iban desde el hilo musical hasta juegos en la tele, pasando por compras y votaciones en programas en directo (Vayatele, 2011), surgen nuevos intentos de potenciar la televisión interactiva.

Vía Digital nace en 1996 como una plataforma por satélite impulsada por Telefónica y RTVE para ser competencia directa de Canal Satélite Digital, una televisión de pago participada por Sogecable y vinculada al grupo Prisa. Desde el principio, Vía Digital apostó claramente por la interactividad.

A través de la plataforma permitía varios servicios en tiempo real: Banca en Casa, a través del cual se podían hacer transferencias, ver movimientos bancarios o comprar entradas por el canal La Caixa; un servicio interactivo sobre el tiempo que informaba, además, del estado de las playas, pistas de esquí, niveles de polen...; un canal de juegos gratuitos y otro de juegos de suscripción, Ludi TV; así como acceso a la información del tráfico, el estado de las carreteras y la información bursátil (Bolsa Activa).

Otros servicios ofertados pasaban por la posibilidad de enviar y recibir correos electrónicos, enviar mensajes cortos a móviles Movistar a través del descodificador, información sobre la Liga Española y la UEFA, una publicidad activa que permitía a los usuarios conseguir regalos o películas para el Canal Palco y el servicio Multipantallas que otorgaba al espectador la posibilidad de elegir el canal desplazando el cursor mediante las teclas de dirección de mando, cambiar de cámara o ver un evento paralelo en otro canal.

Los malos resultados económicos de la plataforma digital provocaron que en 2002 se fusionara con Canal Satélite Digital, y aunque en un primer momento funcionaron como marcas separadas, a partir del 21 de julio de 2003 unificaron contenidos, canales y programación bajo el nombre de Digital+. Con ello, lo que llegó bajo la promesa de revolucionar el sector, tardó relativamente poco en desaparecer, ya que Digital+ decidió no proseguir con su apuesta interactiva.

De forma paralela surgió Quiero TV, la primera y única plataforma de Televisión Digital Terrestre (TDT) de pago de España, propiedad de Endesa (Retevisión), Planeta, BSCH, Iberdrola, Telecom Italia y Media Park (Telefónica), entre otros. Nacida en el año 2000 en un intento de acaparar el mercado de la TDT e intentar hacer competencia a las plataformas digitales por satélite, su principal virtud residía en que sólo era necesario un 
codificador y adaptar la antena existente para disfrutar de su programación.

La nueva televisión se presentaba como "la televisión de internet", ya que permitía navegar en el televisor por medio de un teclado inalámbrico que se suministraba junto al decodificador, del mismo modo que ya se estaba haciendo en Estados Unidos. La interactividad llegó a Quiero TV en junio de 2001 de la mano de un juego, creado por Silicon Artist, que permitía a los abonados enfrentarse entre ellos. Sin embargo, las discrepancias surgidas en el seno de la empresa de Retevisión fueron irreconciliables y acaba con sus emisiones en 2002.

\section{REDES Y TELEVISIÓN, SURGE LAAUDIENCIA SOCIAL}

Casi una década después Internet se vuelve a acercar a la televisión y lo hace de la mano de las herramientas derivadas de la Web 2.0: las redes sociales.

La proliferación en el uso de las redes sociales vinculadas a la televisión, ha puesto de manifiesto que los mensajes televisivos han dejado de ser unilaterales para pasar a ser primero bidireccionales y ahora multidireccionales. Este gran potencial es aprovechado por la televisión para crear nuevos contenidos que dejan de ser pasivos y que se adaptan cada vez más a los gustos y necesidades de las audiencias, de forma que las redes sociales contribuyen a que los ciudadanos participen de forma activa en la configuración del nuevo panorama informativo.

De hecho, son muchos los consumidores que utilizan las redes sociales para obtener recomendaciones y compartir sus propias opiniones sobre sus programas favoritos, convirtiendo el mundo digital en un nuevo espacio en el que debatir (Teixeira: 2012).

Esta nueva relación que se establece entre espectadores y cadenas ofrece a los usuarios la posibilidad de interactuar en tiempo real con el contenido y los espacios publicitarios de todos los soportes: televisión, online y el circuito iWall, una herramienta de software con la que se puede crear entretenimiento interactivo permitiendo que el público envíe textos e imágenes a la pantalla gigante desde sus teléfonos móviles vía SMS, e-mail, Facebook, Twitter o Instagram.

El rápido desarrollo de la Red se ha convertido en un catalizador del cambio de hábitos de uso de la pequeña pantalla, cuyo presente pasa por la emisión digitalizada de sus contenidos. Las cadenas de televisión y sus personajes habituales se suman al poder de las redes sociales a fin de crear auténticas comunidades en Internet, siempre al amparo de la explosión de la participación que proporciona la Web 2.0 (Alonso, 2011:60).

Gracias a las redes sociales la televisión adquiere un nuevo matiz de bidireccionalidad, ya que no sólo ofrece contenidos, sino que escucha a sus espectadores, sus demandas y necesidades, para ofrecerles aquello que buscan y, de esta manera, incrementar sus índices de audiencia, lo que le conduce, de forma paralela, a que el usuario de la Red pase de ser un consumidor de contenidos a participar en la construcción y elaboración de los mismos.

Este fenómeno que desde hace varios años observamos en los periódicos digitales, ha dado ahora el salto a la televisión, la cual, sobre la premisa de que la información es materia prima y la comunicación es el servicio, ha llegado al punto de lo que Lara Tíscar denomina "bloguizado", es decir, el reto de la televisión actual estriba en captar nuevos públicos y convertir a sus audiencias en redes sociales.

"Para ello, los programas de televisión entran en las redes sociales creando una identidad digital para poder relacionarse con su público objetivo, así pues la presencia en Facebook y Twitter es casi obligada para ofrecer información de última hora y servicios a todos aquellos usuarios que se agregan de forma voluntaria a sus cuentas... al medio le interesa crear su propia red social para atrapar y segmentar grupos de población que puedan ser de interés comercial" (Tíscar, 2009: 76).

De hecho, las comunidades virtuales han hecho que el zapping ya no se conciba si no es con un teclado entre las manos, al tiempo que cada vez es más habitual que el público comente en las redes sociales lo que está viendo en la pequeña pantalla. 
En España, tres de cada cuatro usuarios de Twitter publican comentarios mientras ven la televisión y más de la mitad de ellos reconoce que sintoniza un programa concreto por los mensajes que escriben otros usuarios de esta red social, según datos de Media Partnerships Twitter Spain (Salamanca, 2015), que evidencian una inmersión significativa de nuestro país en el consumo multipantalla.

Según datos de un reciente informe de la Fundación Telefónica (2015), más de veintiséis millones de españoles se conectaron a Internet el año pasado. De ellos, el 78,7\% navegó todos los días, porcentaje que se incrementó hasta el $88,9 \%$ en el caso de los jóvenes entre 16 y 24 años. Por su parte, el uso de las redes sociales aumentó tres puntos, hasta el $67,1 \%$, gracias al crecimiento experimentado en la franja de edad comprendida entre 45 y 54 años, que pasa del 43,5 en 2013 al 52,3 por cien en 2014.

Además, nuestro país ha consolidado su liderazgo dentro de los países más relevantes de la Unión Europea en penetración de smartphones, con un $81 \%$ de teléfonos inteligentes sobre el total de móviles, lo que supone diez puntos por encima de la media. Por su parte, la tablet, como dispositivo de acceso, dobla su penetración respecto al año 2014, ya que se encuentra presente en el $28,5 \%$ de los hogares españoles. (AIMC: 2015)

Estos datos propician que la industria televisiva se interese cada vez más por la audiencia social y para ello, las principales cadenas del país han solicitado a sus departamentos multimedia que adapten sus contenidos a las redes sociales con el objetivo de promocionar sus formatos, así como atraer al mayor número de usuarios a través del "muro a muro" que suponen estas plataformas sociales.

En esta carrera por conseguir más audiencia las cadenas televisivas mantienen una encarnizada lucha por tener más amigos y seguidores que la competencia. Así pues, a 27 de abril de 2015 Antena 3TV (@antena3com) es la que más followers posee con 865.999, seguida por RTVE (@RTVE) con 812.799, Telecinco (@telecincoes) con 726.025, La Sexta (@laSextaTv) con 528.831 y Cuatro (@cuatro) con 444.180.

Esta misma tendencia en el número de seguidores la observamos en Facebook, ya que a 1.284.629 personas les gusta Antena 3TV. Le siguen, Telecinco, 1.053.663; Cuatro con 602.987, RTVE, 457.458 y La Sexta, 253.408.

A nivel de impacto social de la televisión es Mediaset quien reina. Según la consultora Global in Media (2015), el grupo de Telecinco acumula un total de 5.446.332 comentarios en Twitter, Facebook e Internet, frente a su competidora directa, Antena 3. De los 9 millones de comentarios realizados por los usuarios en las redes sociales, Mediaset aglutinó el $65 \%$, frente al $21 \%$ acumulado por Atresmedia y el $13 \%$ de RTVE.

Para interactuar con los espectadores, lo más frecuente es recurrir a los hashtags y etiquetas que engloban aquellos temas de los que se quiere hablar. Un claro ejemplo de que la televisión se ve de una forma más participativa y que se disfruta leyendo comentarios y opiniones compartidos en tiempo real, es el acontecido el pasado 14 de abril durante la edición del programa @MasterChef de TVE1, en el que uno de los concursantes se inventó un peculiar plato al que bautizó como "león come gamba". El hashtag con el nombre del plato, \#leoncomegamba, incendió Twitter al generar 83.100 mensajes y una audiencia única social de 538.000 individuos durante la emisión, además de mantenerse como trending topic durante más de 24 horas (Salamanca, 2015).

Las etiquetas también funcionan para crear expectativas y mantener entretenido al telespectador durante la publicidad. Mediaset España acertó en su estrategia publicitaria del estreno de la serie "Homeland", una iniciativa consistente en la emisión exclusiva de bloques cortos hiQuality, con tres spots como máximo, que permite a la audiencia tuitear lo más interesante del capítulo que se está emitiendo. Durante la publicidad, Cuatro propone a sus usuarios comentar las aventuras de la agente Carrie Mathison y el Sargento Brody a través del hashtag \#HomelandEnCuatro.

Esta iniciativa demostró que apenas existe fuga publicitaria, entregando a los anunciantes un rating publicitario medio del $6 \%$ en target comercial, al tiempo que se valora la percepción entre los espectadores a través del hashtag \#3spots, con el cual la compañía obtiene de inmediato el reporte de la opinión de los seguidores sobre esta nueva forma de rentabilizar el contenido. (Alonso, 2014). 


\section{COMUNICACIÓN TRANSMEDIA: MODELOS HACIA LA INTERACTIVIDAD}

Con el firme propósito de atraer a la audiencia y cambiar la relación que tienen con ella, las televisiones han ido incorporando progresivamente técnicas propias de la comunicación transmedia. Henry Jenkins (2003) habla de narrativas transmedia (Transmedia storytelling) para referirse al hecho de narrar una historia a través de distintas plataformas, de tal manera que la comprensión absoluta y el conocimiento profundo de la narración se obtienen cuando se recorren esas múltiples plataformas, soportes y canales.

Aunque es más común encontrar este tipo de narrativas en los productos de ficción, debido a sus características comerciales y de entretenimiento, existen experiencias vinculadas al ámbito informativo. Un claro ejemplo de ello es lab.rtve.es, un laboratorio creado en septiembre de 2010 por el director de rtve.es, Ricardo Villa.

Formado por un equipo de profesionales con conocimientos en periodismo, realización, grafismo y programación, la meta era contar la realidad de una manera distinta y "servir por primera vez de puente entre la televisión, la radio e internet buscando además la integración del usuario y de su participación en la generación de nuevas aplicaciones" (Ava, 2013: 254).

Junto a la creación de docuwebs, infografías y multitud de aplicaciones, lab.rtve.es ha conseguido varios premios de los Lovie Awards, que reconocen los mejores contenidos en Internet: webs, redes creativas, editores de contenido y bloggers amateurs. En concreto, destaca el premio en 2012, en la categoría de Live Events, por "San Fermines interactivos"; y un año más tarde la medalla de bronce en la categoría de televisión por el especial interactivo de Masterchef.

En el Ente público destaca también el proyecto "Las voces de la memoria", en colaboración con la Asociación de Familiares Enfermos de Alzheimer. Utilizando la música como hilo conductor, se evocan los momentos más significativos de la vida de una persona. Fuera de la historia televisada, se creó un canal en Youtube, donde se profundiza en cada historia individual. Inclusive, los usuarios pueden crear su propia Lista de reproducción con aquellos sonidos que marcan la vida de un individuo. Estamos, por tanto, ante comunicaciones transmedia que no sólo buscan la participación, sino que incorporan una finalidad educativa, pedagógica, de servicio público, que muchas veces está ausente en las televisiones privadas.

Independientemente de esos valores, es en la ficción donde tanto las televisiones públicas como las privadas han apostado más por la comunicación transmedia. Para ello, han empleado técnicas diversas como las mencionadas segundas pantallas o second screens, que proporcionan una vía paralela, por la cual el usuario puede pasar a ser un agente activo en la emisión de contenido, llegando a transformar la historia.

Los contenidos generados por los usuarios (User-generated Content-UGC-) expanden el mundo narrativo oficial o lo comentan a través de paratextos. Es, en definitiva, un proceso que implica interacción por parte del usuario. Él es quien decide qué recorrido efectuar y hasta dónde profundizar. Para ello, las redes sociales son imprescindibles. Además, debemos tener en cuenta que las principales aportaciones las encontramos en los contenidos extras para iPad y smartphones, que permiten que el espectador siga consumiendo el producto audiovisual de otra manera, como es el caso del proyecto de second screen de TVE1 para Eurovisión. Mientras se emitían las semifinales o la final, los usuarios podían valorar cada actuación mediante una aplicación.

Las valoraciones se recopilaban permitiendo hacer rankings previos a la puntuación oficial.

"El primer gran ejemplo, que salió un poco extraño, fue aquel en el que preguntamos a la audiencia quién quería que nos representase en Eurovisión y eligieron al Chiquilicuatre. Cuando preguntas a la audiencia a veces no te responde lo que esperas. Lo curioso es que se convirtió en el mayor fenómeno de Eurovisión de los últimos tiempos" (Ava, 2013: 156).

Centrándonos en la ficción televisiva, son las series ‘Águila Roja’ y ‘El Barco’ las que más han popularizado las técnicas transmedia. En la primera se desarrolló una experiencia novedosa de second screen y de gamificación, convirtiéndose en la primera web española que alcanzó un Rose d'Or5 como proyecto interactivo. 
Durante la emisión, los espectadores tenían que encontrar a través de una aplicación web una imagen concreta en el episodio de la serie, que se correspondía con una de las cartas de la colección que se había comercializado para la misma.

"Luego empezamos a llevar todo esto al mundo real. Es decir, retamos a los fans a que jugaran al videojuego en vivo en el propio plató con la participación incluso de los actores de la serie. Venían al plató y hacían lo que denominamos las Fast Play Series" (Ava, 2013: 164), donde el juego se desarrollaba en una hora. La más mediatizada fue la partida en vivo durante el FesTVal de Vitoria.

De esta forma, se va construyendo una comunidad de fans que participan en más iniciativas. Aparecen foros, páginas oficiales de Facebook, etc., y los usuarios van creando sus propios productos. Esa comunidad queda fascinada con el juego online miaguilaroja.com.

"Se intentó que el jugador tomara el rol de gobernador de la villa y adoptara el rol de 'Águila Roja', que pudiera luchar como él cuando le atacaran los demás. Pero no quisimos que luchara contra una máquina, ni contra sus dos amigos, sino que jugaran entre todos los jugadores. Lo que se llama juegos multijugadores masivos" (Ava, 2013: 101).

Las cifras de registro en el juego superaban incluso a otros videojuegos más comerciales, inclusivo en momentos en los que la serie no se estaba emitiendo.

Las estrategias off line se fueron incrementando con el tiempo. Durante el mundial de fútbol, se convocó la "Noche del comisario", donde el público tenía que acudir a una sala con una camiseta roja para poder acceder y poder presenciar, in situ, un gran concierto al que asistieron los principales actores de la serie. Incluso, a través de concursos y live events, muchos usuarios participaron en el rodaje.

Por su parte, 'El Barco' estaba enfocado a adolescentes, fundamentalmente féminas hasta 16 años. En lugar de utilizar Tuenti, red social apropiada para ese target, la productora decidió apostar por Twitter. Crearon los perfiles de todos los personajes de 'El Barco' y escribieron diálogos que no se verían en la televisión.

"Conversaciones que las vayan a disfrutar solamente los fans en Twitter y que tengan un contenido que no puedan tener los demás. Y que sea cocreación. Por ejemplo, que sea la fiesta de Halloween en 'El Barco' y que sean los propios fans los que se disfracen y que luego les demos premios, que puedan venir a plató, que participen en una gymkana, que jueguen al juego online..." (Ava, 2013: 97).

Surgieron así los twittersodios: episodios que se emiten por otro medio de comunicación y que se completan mediante Twitter, añadiendo información y diálogos novedosos, exclusivos para los seguidores. Estos twittersodios se desarrollaban de nueve a diez de la noche, antes de la emisión de la serie en Antena 3.

Relevante también ha sido "The Lost Experience", un ARG (Alternative Reality Game) diseñado por los guionistas de la serie para aumentar el mundo creado en torno a "Perdidos". El juego se desarrolló íntegramente en Internet en todo el mundo:

"No había ganadores ni perdedores, solamente había pistas. En Lost hasta el final los que la seguisteis os daríais cuenta de que quedaron un montón de cosas sin cerrar (...). Los participantes en el ARG de The Lost Experience, pudieron cerrar cosas que no cerraron los demás. Además hubo pistas distintas por cada continente. Entonces la gente que estaba en Australia se coordinaba con la gente que estaba en EEUU y en Europa para poder colaborar y que todos tuvieran la misma información" (Ava, 2013: 99).

Otro ejemplo digno de mención es Alfa 07, una miniserie de la cadena pública germana SWR. En un total de seis episodios se narra cómo el mundo ha llegado a un nivel de control mental como en el libro "1984". Parte de la historia sucede fuera de la tele, en la Red, donde hay piezas audiovisuales que dan pistas y donde se está organizando la resistencia. El proyecto incluye a la radio, donde a través de una serie de podcast que hay que localizar, se narra la historia (Ava, 2013: 158).

Tanto la experiencia alemana como la de la televisión pública española, ponen de manifiesto el gran deseo de enfocar la comunicación hacia narrativas transmedia. Tanto es así, que la Unión Europea de Radiodifusión 
(UER), ha creado, entre otros, el proyecto Treasures, también seis episodios para televisión que tienen como finalidad contar la historia de Europa de una manera diferente. "La localización de la acción varía según el lugar de emisión de la serie. La historia, para hacerla más local, cambia. Además, se utiliza un ARG para hacer el descubrimiento de diferentes contenidos" (Ava, 2013: 159).

\section{APLICACIONES INTERACTIVAS PARA LOS DISPOSITIVOS MÓVILES}

Más allá de su relación con Twitter y en un intento de intensificar la interacción con sus audiencias, Mediaset puso en servicio durante la emisión de "Gran Hermano 14" la aplicación para smartphones y tablets, Appgree (1), un sistema de comunicación creado en España que permitía a los usuarios, reunidos de modo online y unidos por un mismo interés común, ponerse de acuerdo sobre cualquier tema y emitir la opinión mayoritaria de un modo rápido, eficaz y sin intermediarios. El día de su puesta en funcionamiento se realizaron cerca de 228.000 propuestas a las preguntas del grupo por unas 87.000 personas.

Gracias a Appgree, Telecinco introduce un nuevo concepto: el voto social, ya que permitió a sus seguidores incrementar el nivel de interactividad y participación en el reality más longevo y de mayor éxito de la televisión en España, llegando incluso el espectador a "opinar e influir" en el show televisivo. Prueba de ello es que en la gala celebrada el 23 de mayo de 2013 la audiencia pudo participar con su voto social en la nominación de un concursante, el cual acabó siendo nominado por 90.000 personas, el $72 \%$ de los participantes de la votación, y una cifra mayor que los espectadores sociales que participaron en la conversación social en Twitter (72.086), según datos de Tuitele (2013).

Continuando en esta misma senda, y en un intento de alcanzar la tan ansiada televisión interactiva, durante mayo de 2014, y coincidiendo con la emisión del programa "Supervivientes" de Telecinco, el Grupo Mediaset puso en servicio Mitele ON, una nueva aplicación con la que los seguidores del reality podían tomar decisiones a fin de cambiar la vida de los concursantes, así como recibir información exclusiva en tiempo real, seguir el impacto social desde esta segunda pantalla y ver su cara en la pantalla de su televisor.

Esta aplicación se fue desarrollando, ampliando y complementando hasta que en junio, coincidiendo con el Mundial de Fútbol, y cuyos derechos ostentó Mediaset España, el grupo de comunicación y la filial de Publiespaña Integración Transmedia [IT] pusieron en marcha una nueva aplicación para móviles, MIOtv que ofrece al usuario la posibilidad de interactuar en tiempo real con el contenido y los espacios publicitarios de las cadenas que la integran.

Desarrollada en colaboración con TAPTAP Networks (2), MIOtv es una experiencia televisiva inédita cuya principal cualidad es su fácil manejo, ya que el usuario solo tiene que accionar en su teléfono móvil el pulsador MIOtv durante la emisión de un programa frente al televisor, la web o las pantallas iWall.

Si la emisión en ese momento es un contenido de Mediaset España, MIOtv activará tras el "tagueo" (accionar el pulsador) un site especial para determinados espacios o, en su defecto, redirigirá al usuario a microsites genéricos de Telecinco, Cuatro, Factoría de Ficción y Divinity.

MIOtv ofrece una interfaz muy sencilla distribuida a dos columnas en las cuales se localizan parte de los programas y series de ficción que emite el grupo. Al pulsar sobre cualquiera de ellos se accede a información adicional sobre los mismos, retransmisiones, presentadores, actores, personajes y marcas favoritas. De igual forma, ofrece conexión a las principales redes sociales (Twitter y Facebook), así como a la web de los distintos programas.

MIOTv posee, además, un menú desplegable que permite visitar la parrilla televisiva, spots interactivos, el ranking de usuarios de la aplicación y la cantidad de puntos que tienen acumulados, los regalos que se pueden conseguir con esos puntos, un microsite de la Tienda Mediaset donde se pueden adquirir los productos oficiales de programas y series, y un apartado de ajustes que, entre otras funciones, permite recibir notificaciones de las novedades que se van incorporando en el programa. 
Gracias a su tecnología de reconocimiento, MIOtv permite conocer el consumo que los espectadores realizan de la televisión y ofrecerles contenidos a la carta en función de sus preferencias detectadas entre el abanico de posibilidades que ofrecen todos los canales de Mediaset.

A nivel deportivo, y coincidiendo con el Mundial de Fútbol de Brasil 2014, la aplicación permitió seguir cada partido desde distintos ángulos gracias a una multicámara con seis señales que cambiaba la perspectiva de como si se estuviese jugando a la consola: el banquillo visitante o rival, el jugador estrella de cada equipo, la visión táctica o la señal general, además de una central de datos actualizada cada minuto con los resultados, las clasificaciones y los rankings más completos, así como el mejor contenido audiovisual en $360^{\circ}$ : los goles, las jugadas, las mejores fotos y las últimas noticias.

A nivel publicitario, MIOtv garantiza desarrollar proyectos multimedia como el patrocinio, product placement interactivo y la inserción de publicidad mobile en la propia aplicación, la cual permite al anunciante aumentar su engagement con su público objetivo, incrementar y segmentar la atención, cuantificar de forma inmediata y en tiempo real las preferencias de los consumidores y adaptar su comunicación en función de las mismas, así como el e-commerce.

Este producto se sitúa a la vanguardia del $t$-commerce o venta a través de la televisión, ya que el concepto de compra empleando únicamente el mando a distancia se aúna a los nuevos hábitos de de los consumidores, acostumbrados a adquirir productos mediante sus tablets y smartphones. Para ello, solo hace falta accionar el pulsador sobre un spot identificado con la marca MIOtv. De esta forma la aplicación reconocerá el audio y el vídeo generando una landing page (3) o "página de aterrizaje" dotada de un contenido específico o propuesta comercial concreta para el espectador.

Sin llegar al nivel de MIOTv, el grupo Atresmedia también ha iniciado su recorrido en el ámbito de la televisión interactiva gracias a la aplicación Atresmedia Conecta que, desde septiembre de 2014, está disponible para Android, iPhone y iPad y ofrece la posibilidad de disfrutar en directo contenidos exclusivos de las series y programas, así como seguir "eventos" a través de los cuales se pueden recibir vídeos, imágenes, enlaces, pistas y preguntas, entre otros contenidos.

Con un diseño visual e intuitivo, esta aplicación de segunda pantalla tiene por objetivo expandir la experiencia de ver la televisión y para ello ofrece al usuario la posibilidad de saber si la emisión es interactiva para que éste decida si quiere o no participar. Además, favorece los comentarios y la posibilidad de seguir el hilo de los hashtags más utilizados en Twitter sin salir de la aplicación.

\section{A MODO DE CONCLUSIÓN}

El fenómeno masivo de la participación en los programas abre un nuevo campo de acción dentro del ámbito de la televisión interactiva, ya que en cierto modo otorga el control sobre la emisión al usuario, el cual alimenta y retroalimenta la programación.

Según León y García (2002:9), la televisión interactiva puede beneficiarse si se siguen tres estrategias: invertir en la producción de contenidos de gran calidad, aprovechar la interactividad de forma más innovadora y desarrollar las marcas consolidadas en los canales convencionales. Hablaremos de una televisión mucho más personalizada, a la carta, y donde el usuario tendrá un papel de co-creador de contenidos.

Hasta el momento, este fenómeno integrado en el televisor, una de las soluciones realizables con el estándar HbbTV, no ha tenido especial trascendencia en España y eso a pesar de que el crecimiento de la adquisición de televisores interconectados supera el $16 \%$. Ello es debido a que el target del consumidor de televisión convencional es muy variado y no siempre domina la tecnología, pero también a que se trata de un sistema poco explotado por los canales, de manera que muchos usuarios desconocen que disponen de contenidos añadidos.

Por este motivo, se hace patente la necesidad de producir un contenido que despierte interés entre los usuarios 
y que les aporte un valor añadido. Esta capacidad de llenar el timeline de las cadenas con una programación variada, de calidad y competitiva, será la clave del éxito o fracaso, ya que la audiencia y sus gustos continúan siendo la única ley que se impone en el mercado, por encima de innovaciones tecnológicas.

\section{REFERENCIAS BIBLIOGRÁFICAS}

\subsection{Libros}

AVA (2013): Profesionales audiovisuales ante el nuevo escenario transmedia. Colección: Andalucía Promoción Audiovisual, Nº 11. Fundación Audiovisual de Andalucía, Junta de Andalucía, Sevilla.

\subsection{Artículos en revistas}

ALONSO, M. (2014). Audiencia social: el telespectador comienza a participar en los contenidos televisivos. Ámbitos. Revista Internacional de Comunicación, 25. (http://ambitoscomunicacion.com/2014/audiencia-social-eltelespectador-comienza-a-participar-en-los-contenidos-televisivos/\#more-1117)

JENKINS, H. (2003). Transmedia Storytelling. Moving characters from books to films to video can make them stronger and more compelling. Technology Review, 15.

http://www.technologyreview.com/news/401760/transmedia-storytelling/

LEÓN, B. Y GARCÍA, J. A. (2002): Los retos de la implantación de la televisión interactiva a la luz de su propia historia. Zer, Vol.7, 13. (http://www.ehu.eus/ojs/index.php/Zer/article/view/6024/5706)

TíSCAR, L. (2009). La nueva esfera pública. Los medios de comunicación como redes sociales. Telos, 76, 128131. [DOI: 10.4185/RLCS-64-2009-806-71-8].

\subsection{Webs}

AIMC (2015). Resumen general de resultados EGM. (www.aimc.es/-Datos-EGM-Resumen-General-.html) [Consulta 15 abril 2015].

FUNDACIÓN TELEFÓNICA (2015). La Sociedad de la Información en España.

(http://www.fundaciontelefonica.com/2015/01/21/sie14-informe-sociedad-informacion-espana-pais-conectadoeuropa/) [Consulta 22 enero 2015].

GLOBAL IN MEDIA (2015). iRedes 2015:resumen del impacto en redes sociales.

(http://www.globalinmedia.com/iredes-2015/) [Consulta 16 abril 2015].

PÉREZ, J. (2011). Historia de la televisión interactiva (http://es.slideshare.net/joanakin/historia-de-la-televisininteractiva) [Consulta 14 septiembre 2011].

SALAMANCA, A. (2015). Tres de cada cuatro usuarios de Twitter comentan mientras ven la televisión.

(http://www.elmundo.es/television/2015/04/27/553e1a7c268e3e72498b456b.html) [Consulta 27 abril 2015].

TEIXEIRA, S. (2012). Las redes sociales influyen a la hora de seleccionar nuestra programación televisiva. Aula Tic Pymes (http://www.usc.es/atpemes/spip.php?page=imprimir\&id_article=3888) [Consulta 23 mayo 2014].

TUITELE (2013). Un año de televisión social en España.

(http://informes.tuitele.tv/emailing/Tuitele_1_a\%C3\%B1o_tv_social_en_Espa\%C3\%B1a.pdf) [Consulta 15 agosto 2013].

VAYATELE (2011). HbbTV: Cómo es el nuevo intento de televisión interactiva. (http://www.vayatele.com/nuevastecnologias/hbbtv-como-es-el-nuevo-intento-de-television-interactiva) [Consulta 8 julio 2014]. 
(1) Desarrollado por compañía madrileña MoonCastTV S.A. Appgree es un juego de palabras en inglés que se presta para decir «aplicación para el acuerdo». En su primera semana alcanzó el primer puesto del top 10 en descarga de aplicaciones gratuitas de iPhone.

(2) La mayor red independiente de publicidad móvil en España y la primera red Premium del mercado. Fundada en marzo de 2010, su objetivo es maximizar el engagement del usuario final y la monetización del contenido de los editores asociados.

(3) Es la página web a la que una persona llega después de haber pulsado en el enlace de algún banner o anuncio de texto situado en otra página web o portal de Internet. En la mayoría de los casos, esta web es una extensión del anuncio de promoción donde se explica detalladamente la oferta del producto o servicio que se está promocionando a través de una carta de ventas.

\section{BREVE SEMBLANZA DE LAS AUTORAS}

Marián Alonso González es Doctora en Comunicación por la Universidad de Sevilla (2008) con una Tesis Doctoral que versa sobre el cambio tecnológico de $A B C$ de Sevilla. Técnico de Comunicación en la Dirección de Comunicación y Gabinete de Presidencia de ADIF, compagina su actividad investigadora con su labor docente como profesora asociada de la Facultad de Comunicación impartiendo clases de Redacción Periodística Audiovisual.

María José García Orta es Doctora en Periodismo por la Universidad de Sevilla, es profesora asociada en el Dpto. de Periodismo II de dicha Universidad y miembro del Grupo de Investigación Medios, Políticas de Comunicación y Democracia en la Unión Europea. Su actividad científica la ha desarrollado en el ámbito de la propaganda y en otras parcelas de conocimiento, entre las que destacan la comunicación política electoral y las redes sociales.

Ámbitos. Revista Internacional de Comunicación, n.35, edición de invierno, 2016-2017. Recibido: 16/09/2016

Aprobado: 24/11/2016 